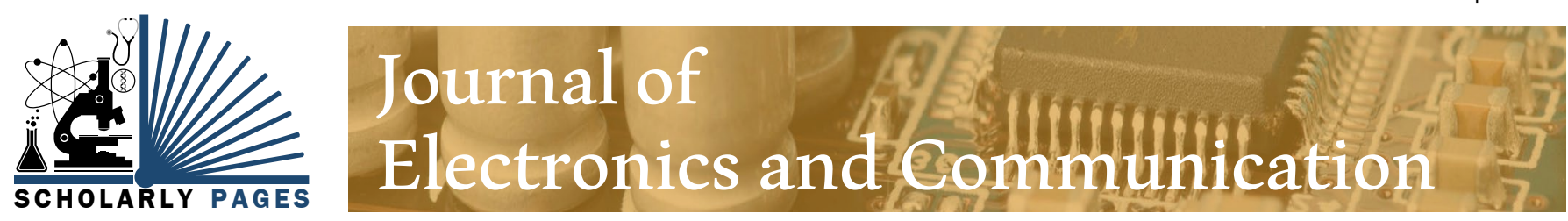

Review Article

\title{
Nikola Tesla's Free Electricity Electronic Circuit
}

\section{Manu Mitra*}

\section{Electrical Engineering Department, University of Bridgeport, Connecticut, USA}

\begin{abstract}
Nikola Tesla was an inventor who is best known for his contributions for the design for generating alternating current, Electricity supply system etc. He obtained around three hundred patents worldwide for his inventions and some are hidden in patent archives. One of the patents that discussed providing free electricity is "Method of utilizing radiant energy" was successfully demonstrated but was never completed.

After careful reading Tesla's articles and patent application; design of electronic circuitry for the free electricity can be constructed. Although, there is no obvious reason to generate kilowatts of power through simple circuitry. This paper gives insight and review of Nikola Tesla's free electricity invention.
\end{abstract}

Keywords

Nikola tesla, Radiant energy, Electronic circuit, Electronics, Free electricity, Static electricity

\section{Introduction}

One of the Nikola Tesla's attempt to provide everyone in the world with free energy was his World Power System, a method of broadcasting electrical energy without wires, through the ground that was never finished, but his dream of providing energy to all points on the globe is still alive today [1].

Tesla's intent was to condense the energy trapped between the earth and its upper atmosphere and to transform it into an electric current. He pictured the sun as an immense ball of electricity, positively charged with a potential of some two hundred billion volts. On the other hand, earth is charged with negative electricity. The tremendous electrical force between these two bodies constituted, at least in part, what he called cosmic energy. It varied from day to night and from season to season but it is always present [2].

In 1931 Tesla announced in The Brooklyn Eagle that "I have harnessed the cosmic rays and caused them to operate a motive device. More than 25-years-ago I began my efforts to harness the cosmic rays and I have succeeded. Electric power is everywhere present, in unlimited qualities. This new power for the driving of the world's machinery will be derived from the energy which operates in the universe, without the need for coal, gas, oil, or any other fuel".
The real reason Nikola Tesla for interest in free energy was he learned that there was warming of the planet caused by natural and manmade sources of atmospheric pollutants. Tesla may have been first human to learn that now it's called "Global Warming" and "Greenhouse Effect".

After many years, in 2003 a company named Tesla Motors was incorporated by Martin Eberhard and Marc Tarpenning named after Nikola Tesla, the company was to commercialize electric vehicles using an AC motor which was built on the foundations of a design that Nikola designed in 1882. In February 2004 Elon Musk, co-founder of PayPal, invested in Tesla and became the Chairman of the company. Tesla Motors have created several electric cars all of which save on energy costs and help the environment by producing zero emissions. Instead of gas stations, Tesla cars can be recharged by using any of a number of free charging stations, Tesla owners can simply plug their cars in and in around 20 minutes completely recharge their car, shaking up the motor in-

*Corresponding author: Manu Mitra, Electrical Engineering Department, University of Bridgeport, Connecticut, USA, E-mail: manu.ieee@gmail.com

Received: October 26, 2017; Accepted: March 03, 2018; Published online: March 05, 2018

Citation: Mitra M (2018) Nikola Tesla's Free Electricity Electronic Circuit. J Electron Commun 1(1):1-6

Copyright: (C) 2018 Mitra M. This is an open-access article distributed under the terms of the Creative Commons Attribution License, which permits unrestricted use, distribution, and reproduction in any medium, provided the original author and source are credited. 
dustry and upsetting fuel providers. The journey to $\mathrm{Ni}$ kola Tesla's dream of free energy for everyone has come to another major checkpoint. Elon Musk announced Tesla Energy, a battery system that stores electricity from solar panels and can power homes and businesses. There are two products, the Power wall and the Power pack. The Power wall comes as a $10 \mathrm{kWh}$ or $7 \mathrm{kWh}$ unit and can create networks that can be used to store redundant power or ultimately enable homes to eliminate the need to be connected to the power grid $[3,4]$.

\section{Radiant energy}

The electrical, radiant, energy throughout the universe, which Tesla referred to and would use to provide power wirelessly of all the inventions and discoveries of Nikola Tesla, stood out with greater potential benefit to the whole of humanity and his discovery of Radiant Energy. Only after conducting exhaustive experimental trials for three years, did Tesla announce this discovery in a paper published in December, 1892, entitled "The Dissipation of Electricity" [5].

Radiant Energy is always there in our atmosphere. Radiant energy includes solar radiation from the sun, as well as cosmic rays from the stars. New and varied free energy devices harness this power to produce electricity [6].

\section{Static electricity}

We all have been subject to static electrical charges after we do our laundry or if we scuff our shoes on a rug too much. There are ways to harness it [6].

\section{Working Principle}

From the electric Potential that exists between the elevated plate (positive) and the ground (negative), energy builds in the capacitor, and, after "a suitable time interval", the accumulated energy will "manifest itself in a powerful discharge" which can do work. The capacitor, says Tesla, should be "of considerable electrostatic capacity", and its dielectric made of "the best quality mica", for it has to withstand potentials that could rupture a weaker dielectric.

Tesla gives various options for the switching device. One is a rotary switch that resembles a Tesla circuit controller. Another is an electrostatic device consisting of two very light, membranous conductors suspended in a vacuum. These sense the energy build-up in the capacitor, one going positive, the other negative, and, at a certain charge level, are attracted, touch, and thus fire the capacitor. Tesla also mentions another switching device consisting of a minute air gap or weak dielectric film which breaks down suddenly when a certain potential is reached.

A certain device consists of a long electric wire (about $100 \mathrm{~m}$ ) that is suspended a few meters above the ground (possibly by a tree or wooden fence), and not in contact with any other conductors.

\section{Static power device}

Below are some points to be consider for Tesla's cable for a long wire

- The wire must be kept from touching any conducting material. The length and height of the wire determines the accumulation/rate of the charge, and the wire must remain stiff at all times.

- Once laid out, the wire must be attached to a few different components such as a spark plug, a condenser,

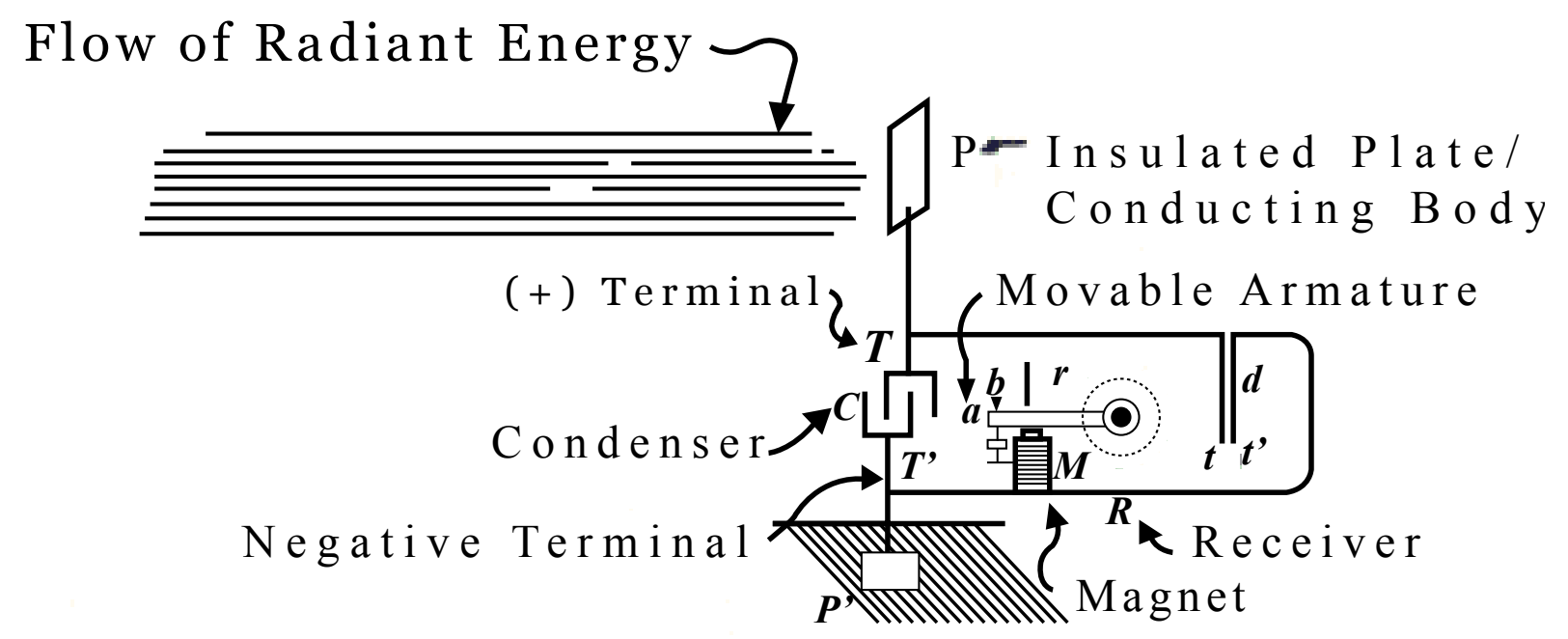

Grounded Plate

Figure 1: Tesla's free energy receiver. 


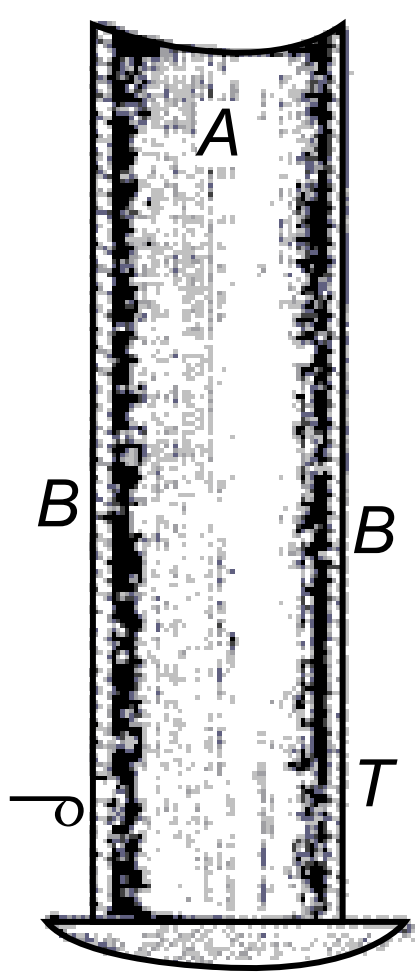

Figure 2a: Tesla's cable for generating electricity from thin air. $A \rightarrow$ medium with little energy; $B_{1}, B \rightarrow$ ambient medium with much energy; $\mathrm{O} \rightarrow$ path of energy.

and ignition coil, a diode, and a battery, at specific positions and earthed at certain points as well.

- The wire will absorb electrostatic charges from the atmosphere which is then guided by the other components to the battery which stores these charges.

- This stored charge can be used anytime to produce electric current. This process is a continuous one, as the wire keeps accumulating charges all the time.

- The extent of the amount of electricity produced depends on the weather as well. A windy day, week or month will increases the efficiency, whereas a humid atmosphere reduces the efficiency of the system.

- This method is similar to the method for collecting T.V. and Radio waves for electricity $[7,8]$.

Nikola Tesla's patent "Apparatus for the utilization of Radiant Energy" (US000685957) shows that how radiant energy can be captured and can be converted to electricity (Figure 1).

According to Nikola Tesla, energy cannot transfer across it except through a channel $\mathrm{O}$, in this enclosure a medium were maintained which would have little energy, and that on the outer side of the same there would be the ordinary ambient medium with much energy.

Under these assumptions the energy would flow through the path $\mathrm{O}$, as indicated by the arrow, and might

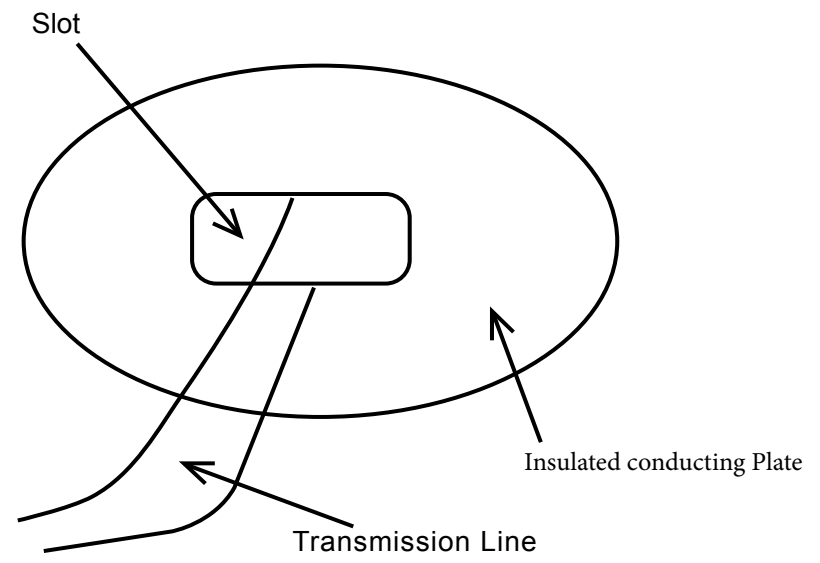

Figure 2b: Concept of Tesla's plate antenna for capturing radiant energy [9]

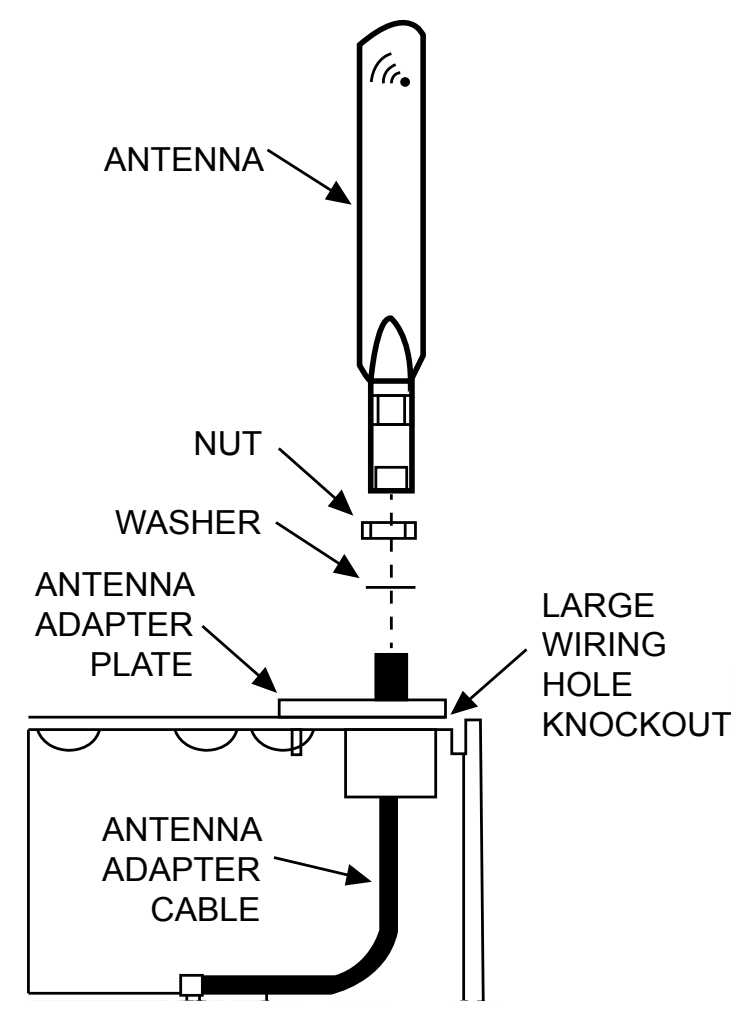

Figure 3: Conceptual design of tesla's free electricity through capturing radiant energy.

then be converted on its passage into some other form of energy. That could produce artificially such a "sink" for the energy of the ambient medium to flow in be enabled to get at any point of the globe a continuous supply of energy, day and night [5] (Figure 2a, Figure 2b and Figure 3).

\section{Circuit Diagram}

A circuit diagram was created based on the Nikola Tesla's "Method of utilizing radiant energy" Figure 4 shows the construction of a circuit diagram using four diodes and a capacitor which is a bridge rectifier. 


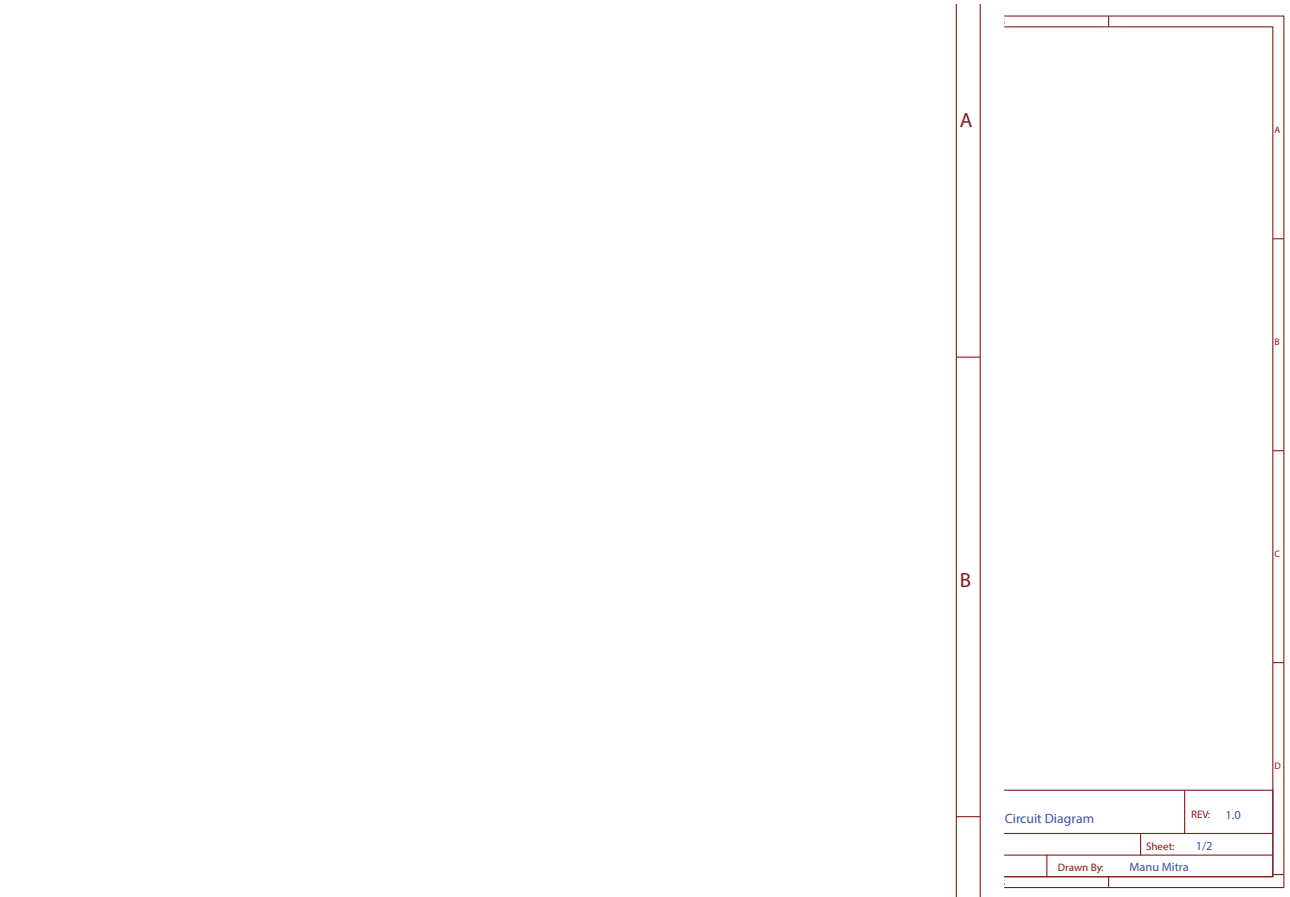

Figure 4: Circuit diagram of utilizing radiant energy [10].

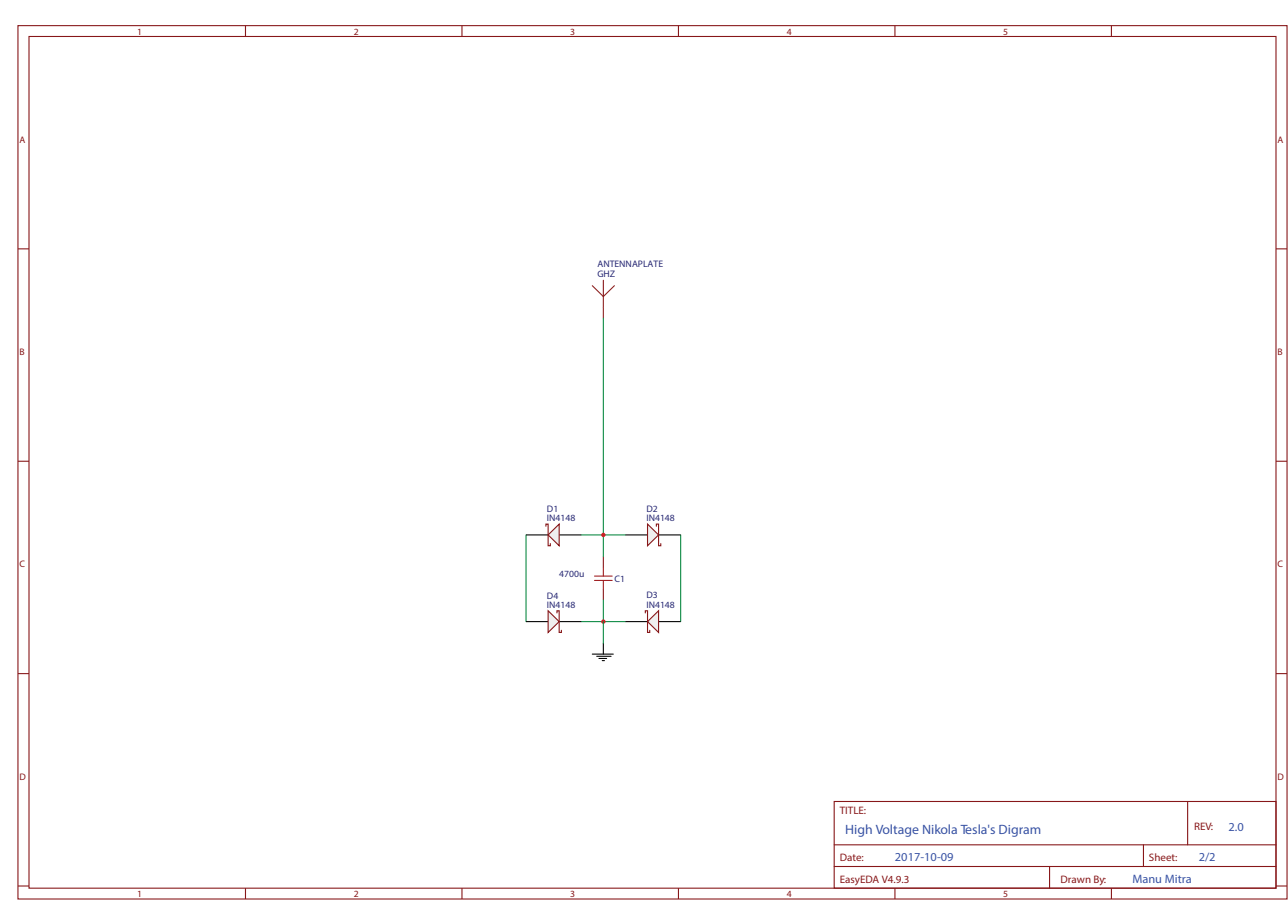

Figure 5: High voltage circuit diagram of utilizing radiant energy using zener diodes (not tested).

For the demonstration purpose four diodes (RL 207) and one capacitor (200v-220 micro) and one insulated plate antenna have been used. Figure 5 shows the construction of a circuit diagram using four Zener diodes and a capacitor which is a bridge rectifier.

For the demonstration purpose four Zener diodes (IN 4148) and one capacitor (4700 micro) and one insulated plate antenna have been used.
In Figure 6 the long coaxial cable acts as an antennae and a capacitor and as it builds up charge, it discharges spark that jumps the gap, is then stepped down by the coil, and then pulsed to the battery through the use of the capacitor.

The long wire captures radiant energy, as the charge builds up and jumps the gap, spark (longitudinal waves) it is then stepped down in the coil, (The coil in the car 


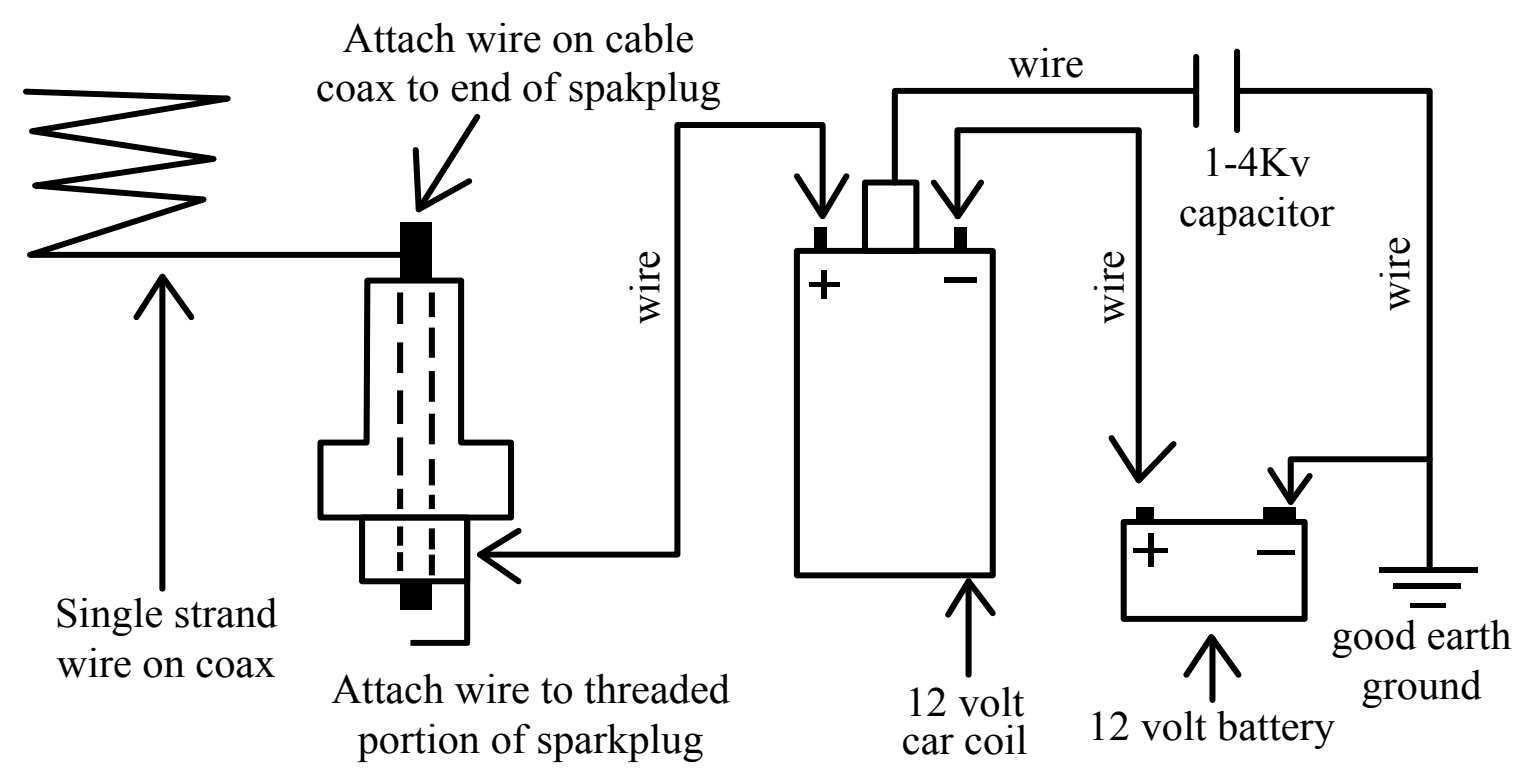

Figure 6: Technical circuit diagram to charge a battery for example an inverter as per Nikolas Tesla's invention [Image Credit 11].

was invented by Tesla and is a small Tesla coil,) it is then charging the battery where it is transformed via a square or PWM wave at low frequency and is the reactive power that charges the battery.

\section{Coax cable}

The end of the coax is taped off and the end that is attached to the spark plug the wires are twisted together.

The rate of charge in the wire that is oscillating in nature at one frequency and the discharge frequency in sharp DC transient pulse to the battery that is at its own rate. Pulse rate would almost look like a triangle wave flattened off and would follow the gravitational wave frequency of about $8 \mathrm{KHZ}$.

\section{Applications, Advantages and Disadvantages}

\section{Applications}

At this time, it may not generate Kilowatts of power for household or industrial purpose but if the circuit is improved by using Zener diodes with respective resistors, capacitors (as shown an example in the Figure 5) and if broadness of Antenna and height is increased then there is enough power can be generated to charge an ordinary invertor battery continuously.

If properly designed and connected to the invertor it can light one ordinary tube light, one ceiling fan, one laptop and one wireless modem which can run on invertor and invertor charges continuously with the radiant energy.

\section{Advantages}

The major advantage is that there is no recurring fee for electricity. Only one time payment for Tesla's Antenna, Tesla's cable and one can generate significant amount of power for charging the invertor.

Few of the advantages of using Nikola Tesla's method are:

- Clean, economical, safe and convenient source of energy.

- No tailpipe emissions.

- Pollution free power generation.

- Simple construction, mature technology and easy maintenance once it gets into production.

- No manual work necessary during generation.

- Energy available all year around.

- No fuel transportation problem.

- No consumption of any fossil fuel which is non-renewable source of energy.

- Produces no waste or greenhouse gases.

- Lower maintenance.

- Maximum utilization of energy.

- Works better when recharging battery.

- Most importantly it's Free, sustainable and can also provide electricity to remote places.

\section{Disadvantages}

One of the disadvantages is if one has to generate more power; broadness of antenna, and height of receiver should also be increased.

At this time thousand watts of power cannot be generated from the given above simple circuit. But given the fact that if there is a tower that can be as high as Warden- 
clyffe Tower then some significant amount of continuous power can generated as multiple cables can deployed and Antenna size can also be increased thus increasing onetime expense.

Few of the disadvantages stated:

- Onetime expense.

- For higher power generation, towers need to be installed.

- It's not always predictable- windy days may be higher efficiency and sometimes it may have lower efficiency.

\section{Conclusion}

In this review paper of Nikola tesla's free electricity are

- Free Electricity circuitry can be designed based on the Nikola Tesla's patent For high voltages Zener Diodes can be used provided circuit uses proper resistors and capacitors.

- Using Nikolas Free Electricity one can generate electricity to charge an ordinary invertor battery continuously.

- Significant amount of power can be generated if it is used at higher height like towers and multiple antennae and cables can deployed, thus increasing efficiency and continuous power generation.

- Higher level of continuous Electricity can be achieved if height is higher (like Wardenclyffe Tower) assuming broadness of antenna is also increased.

- If some of the Towers like Wardenclyffe are installed then significant amount of electricity can be generat- ed and can be used for household and small industries.

- For high power electricity large and broad insulated conductive antenna with high coaxial cables which can be installed on the towers for commercial purpose. Hence significant amount of continuous electricity can be generated to charge battery continuously.

\section{Acknowledgment}

Author would like to thank Prof. Navarun Gupta, Prof. Hassan Bajwa and Prof. Linfeng Zhang for their academic support. Author also thanks anonymous reviewers for their comments.

\section{References}

1. Free Energy.

2. Bruce AP (2013) Nikola Tesla free energy: Unraveling greatest secret.

3. Adam MB (2015) The journey to Nikola Teslas dream of free energy for everyone. Tech Bubble Technologies.

4. Free Energy-Fact or Fiction?

5. Tesla's Discovery of Radiant/Dark Energy.

6. Farmer AC How to get or make free energy \& save on electricity bill. Slide share.

7. Justin Deschamps (2013) Stillness in the Storm. An Agent for Consciousness Evolution.

8. George T (1901) Free Energy Receiver. The Lost inventions of Nikola Tesla.

9. Antenna Model.

10. Circuit Simulation.

11. Emmett Butler (2004) Radiant Energy Aerial Capture (REAC) \& Transformation Device. KEELYNET. 